Military Technical College

Kobry El-Kobbah

Cairo, Egypt

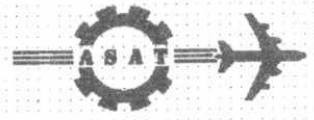

$10^{\text {th }}$ International Conference

On Aerospace Sciences\&

Aviation Technology

\title{
Fuzzy Data Association Filter (FDAF) For Maneuvering Multitarget Tracking.
}

\author{
ASHRAF M. ANWAR* and AHMED A. BAHNASAWI*
}

\begin{abstract}
.
This paper presents a new algorithm for Manoeuvring Multitarget Tracking. The suggested algorithm solves the interrelated tasks of data association and state estimation in one combined algorithm. The new algorithm is based on fuzzy cluster means algorithm to solve the data association problem, and an adaptive Kalman filter for maneuvering multitaget tracking. To demonstrate the effectiveness of the proposed algorithm to perform data association and state estimation in multitarget tracking in high noisy measurement, an example of four-dimensional tracking system is considered. A scenario of two targets moving together at near distance and then making high maneuver is considered. The performance is evaluated using Monte Carlo simulations and the results are reasonable.
\end{abstract}

Keywords: data association; tracking systems; fuzzy logic; multisensor multitarget tracking; intelligent tracking.

\footnotetext{
"Graduate student, Dpt. Of Elect. and Comm., M.T.C, Cairo, Egypt.

"Professor, Dpt. Of Elect. and Comm., Fac. Of Eng. Cairo University, Guiza, Egypt.
} 


\section{1-Introduction.}

Ordinary tracking problems involve processing measurements (e.g., range and azimuth observed by a sensor) from a target of interest and producing, at each time step, an estimate of the target's current position and velocity vectors. Uncertainties in the target motion and in the measured values, usually modeled as additive random noise, lead to corresponding uncertainties in the target state.

A common and versatile approach to such problems involves assuming that the state dynamics and the measurements are both corrupted by additive, white Gaussian noise; the solution is then the Kalman-Bucy filter [1-3]. The parameters that determine tracking performance in such a filter are the system matrices in the equations describing target state dynamics and measurements, which will be considered fixed for the purposes of this discussion, and the covariance matrices of the process and measurements noises, which specify the uncertainties in target motion and measured values, respectively.

In many tracking problems, particularly those arising in surveillance, there is additional uncertainty regarding the origin of the received data, which may (or may not) include measurements from the targets of interest, interfering targets, or random clutter (false alarms). This leads to the problem of data association or data correlation, which has been on a number of fronts [5-16]. In this situation, tracking performance depends not only upon the noise covariances, but also upon the amount of uncertainty in measurement origin.

One can broadly divide the various aspects of a multitarget-tracking (MTT) algorithm into the interrelated tasks of data association and state estimation. Targets tracking involve: 1) the evaluation of a measure of how likely it is that a particular measurement originated from a target and 2) the update state of the target, if the measurement is assigned to the target by (the data association logic). The data association part of a multitarget-tracking algorithm is the decision process of linking together measurements of a common origin (i.e. a target or false alarms) so as to maximize a certain global performance measure, while satisfying certain feasibility condition (e.g., each measurement is associated with only one origin).

In the literature, there are two main categories of data association for MTT systems: algorithmic and nonalgorithmic. The algorithmic category is based on nearest-neighbor and all-neighbor techniques. The nonalgorithmic category is based on neural network and fuzzy logic techniques. [22]

Fuzzy logic techniques are well suited to model decision making processes [21]. Application of the fuzzy logic to the data association problem provides an approximate solution, and the results are subjected to the number of input variables, number of linguistic variables, the membership function, and the accuracy of the rules. In fuzzy clustering, each data point can be associated with more than one cluster with some degree of membership. The membership degrees are determined in a way to minimize or maximize a function. Recently, fuzzy clustering has been applied to data association and target identification. [20-23,25]

However, in the multitarget case where association and estimation are coupled, one needs a systematic estimator that automatically adapts itself, and this is the major contribution of the present work.

The remainder of this paper is organized as follows: section 2 formulates the tracking problem. Fuzzy cluster means algorithm is reported in section 3. The proposed Fuzzy Data Association Filter is presented in section 4. The simulation results are illustrated in section 5 . Finally the conclusion is adopted in section 6. 


\section{2-Problem formulation.}

The mutitarget-tracking problem may be briefly stated as follows: Given a large number of close measurements for a group of targets. It is difficult to determine precisely which target corresponds to each of the closely --spaced measurements.

It is assumed that the only measurements available are range $R$ and bearing angle $\theta$, and filtering is performed in the rectangular coordinates (two-dimensional) so that the measurements must first be transformed. The transformation equations are

$$
\begin{aligned}
\mathrm{X}(\mathrm{t}) & =\mathrm{R} \cos (\theta) \\
\mathrm{Y}(\mathrm{t}) & =\mathrm{R} \sin (\theta)
\end{aligned}
$$

The state equation for maneuvering target model, discretized over time intervals of length $T$, is

$$
\left.\begin{array}{rl}
\mathrm{X}(\mathrm{k}+1) & =\mathrm{A} \mathrm{X}(\mathrm{k})+\mathrm{B}[\mathrm{G}(\mathrm{k})+\mathrm{w}(\mathrm{k})]
\end{array}\right]
$$

$\mathrm{x}(\mathrm{k}), \mathrm{y}(\mathrm{k}), x(k)$ and $y(k)$ represent the target positions and speeds in the $\mathrm{X}$ and $\mathrm{Y}$ directions, respectively.

$$
A=\left[\begin{array}{llll}
1 & T & 0 & 0 \\
0 & 1 & 0 & 0 \\
0 & 0 & 1 & T \\
0 & 0 & 0 & 1
\end{array}\right]
$$

(the state transition matrix)

$$
B=\left[\begin{array}{ll}
T^{2} / 2 & 0 \\
T & 0 \\
0 & T^{2} / 2 \\
0 & T
\end{array}\right]
$$

(the input matrix), where $\mathrm{T}$ is the sampling interval.

$$
G(k)=\left[\begin{array}{l}
g_{x}(k) \\
g_{y}(k)
\end{array}\right]
$$

(the acceleration input vector of the maneuvering target at time $\mathrm{k}$ ).

$$
w(k)=\left[\begin{array}{l}
w_{x}(k) \\
w_{y}(k)
\end{array}\right]
$$

(the input process noise vector with white zero mean)

The measurement equation is modeled as:

$$
Z(k)=H(k) X(k)+V(k)
$$


Where $\quad Z(k)=\left[\begin{array}{llll}z_{x}(k) & z_{\dot{x}}(k) & z_{y}(k) & z_{y}(k)\end{array}\right]$

(the observation vector at time $\mathrm{k}$ with dimension $\mathrm{m}$ )

$\mathrm{H}$ : Identity matrix (it was assumed that both position and velocity in the $X$ and $Y$ directions are measured)

$V(k)$ : (measurement noise vector with white zero mean).

The plant noise and the measurement noise sequences are assumed to be uncorrelated; zero mean Gaussian sequences with the corresponding covariance matrices $Q, R$ :

$$
\begin{aligned}
& E\left[w(k) w(j)^{\top}\right]=Q_{k} \delta_{k j} \\
& E\left[v(k) v(j)^{\top}\right]=R_{k} \delta_{k j} \\
& E\left[w(k) v(j)^{\top}\right]=0
\end{aligned}
$$

And $\delta_{k j}$ is the Kronecker delta function.

The problem treated here is how to construct the target states estimate by using the uncertain measurements originating from the targets in track. In particular the estimate is to be computed when tracking maneuvering multitargets.

\section{3-Fuzzy clustering means algorithm.}

The most widely used clustering algorithm is the fuzzy clustering means (FCM) algorithm developed by Bezdek [26]. This section introduces the FCM algorithm, which will be used for measurements -to-track association (correlation) The goal of any fuzzy clustering algorithm is to classify the data into a number of clusters (groups). The clustering algorithms produce a degree of membership for each data point in each cluster. Unlike conventional clustering, which involves a partitioning of objects into disjoint clusters. fuzzy clustering allows a data point $x$ to have a partial degree of membership in more than one set $[27,28]$. A fuzzy set $A$ in a collection of objects $X$ is defined as $[27,28]$

$$
A=\left\{\left(X, u_{A}(x)\right), x \notin X\right\}
$$

Where $u_{A}(x)$ is the degree of membership function of data point $x$ in fuzzy set $A$. Given a number of data points, it is required to group (cluster) the data into clusters according to some similarity measure. Let $c$ be an integer, which represents the number of clusters with $2 \leq c \leq n$, where $n$ is the number of data points. Define $U$ as partition matrix of elements $u_{i j}(i=1,2, \ldots, c, j=1,2, \ldots, n)$ which represents the degree of membership of data points $j$ in fuzzy cluster $i$, such that

$$
\begin{gathered}
\mathrm{u}_{\mathrm{ik}} \in[0,1] \quad 1 \leq \mathrm{i} \quad \leq \mathrm{c}, \quad \quad 1 \leq \mathrm{k} \leq \mathrm{n} \\
\sum_{i=1}^{c} u_{i k}=1 \quad \forall k \\
0<\sum_{k=1}^{n} u_{i k}<n \quad \forall i
\end{gathered}
$$


Define $J_{m}$ as the sum of the squared errors weighted by the $m^{\text {th }}$ power of the corresponding degree of membership, i.e.,

$$
J_{m}(U, v)=\sum_{k=1}^{n} \sum_{i=1}^{c}\left(u_{i k}\right)^{m}\left(d_{i k}\right)^{2}
$$

where

$$
\left(d_{i k}\right)^{2}=\left\|x_{k}-v_{i}\right\|^{2}
$$

$x_{k}$ is a data piont and $v_{i}$ is a cluster center, and $/ / . / /$ is induced norm, $m$ is a real number $€[1, \infty)$ called the fuzzification constant (or weighting exponent). The degree of membership will be established by minmizing the sum of the squared errors weighted by the corresponding $\mathrm{m}^{\text {th }}$ power of the degree of membership. The goal of fuzzy clustering algorithm is to determine the optimum degree of membership $u_{i k}$ and the optimum fuzzy $v_{i}$ such that the sum of the squared errors $J_{m}$ is minimum. The result is given by [26]

$$
\begin{gathered}
u_{i k}=\frac{1}{\left[\sum_{j=1}^{c}\left(d_{i k} / d_{j k}\right)^{2(m-1)}\right]} \quad \forall i, k \\
v_{i}=\frac{\sum_{k=1}^{n}\left(u_{i k}\right)^{m} x_{k}}{\sum_{k=1}^{n}\left(u_{i k}\right)^{m}} \quad \forall i
\end{gathered}
$$

Where (3.7) is valid for a fixed $V\left(V=v_{1}, V_{2}, \ldots . v_{c}\right)$, and $(3.8)$ is valid for a fixed $U$. In MTT systems, $c$ is the number of targets, $n$ is the total number of received measurements, $x_{k}$ is the $s$-dimensional measurement vector $(k=1,2, \ldots, n)$ and $v_{i}$ is the $\mathrm{s}$-dimensional predicted vector for target $\mathrm{i}(\mathrm{i}=1,2, \ldots, \mathrm{c})$. The fuzzy $\mathrm{c}$-means clustering algorithm or the Picard algorithm is guaranteed to converge to a local minimum [29].

It is worth to mention that the fuzzification constant $m$ plays an important role. It reduces the influence of noise when computing the degree of membership (3.7) and the cluster center (3.8). The weighting exponent $m$ reduces the influence of a small $u_{i k}$ (for data that are faraway from the cluster centers) compared to a large $u_{i k}$ (for data that are closed to the cluster centers). As $m$ increases, its influence becomes stronger [23].

4- Proposed Fuzzy Data Association Filter (FDAF).

The state estimate of target $i$ is updated based on the new measurement by: $\hat{x}_{i}(k+1 / k+1)=\hat{x}_{i}(k+1 / k)+K_{i}(k+1) v(k)$

where $\quad v_{i}(k)=\sum_{j=1}^{N_{v}} u_{i, j}(k) v_{i, j}(k) \quad i=1,2, \ldots ., c$

is the weighted innovation, which uses all the validated measurements $\left(N_{v}\right)$.

$u_{i, j}(k)$ is the degree of membership of measurement $j$ to target $\mathrm{i}$ at time $\mathrm{k}$ as obtained by (3.7) 
$v_{i, j}(k)=z_{j}(k)-\hat{z}_{i}(k / k-1)$

is the innovation due to new measurement

$K_{i}(k+1)$ : is the standard Kalman filter gain.

If we proceed by the same manner as the well known Probabilistic Data Association Filter technique [3,9]

The updated covariance can be obtained as:

$p_{i}(k+1 / k+1)=p_{i 1}(k+1 / k+1)+p_{i 2}(k+1)$,

$p_{i 1}(k+1 / k+1)=\left[I-K_{i}(k+1) H_{i}(k+1)\right] p_{i}(k+1 / k)$

is the standard covariance update equation, and

$p_{i 2}(k+1)=K_{i}(k+1)\left[\sum_{j=1}^{N} u_{i j} v_{i j}(k+1) v_{i j}(k+1)-v_{i}(k+1) v_{i}(k+1)\right] K^{\prime}(k+1)$

The matrix $P_{i 2}(k+1)$ shows the effect of the measurements that did not originate from the object in track by increasing the covariance of the estimate. This follows from the fact that the matrix $P_{i 2}(k+1)$ is positive semi definite [9]. The flow diagram of the (FDAF) is shown in Fig. 1 
Enter prior estimate $\hat{x}_{0}^{-}$and its covariance $p_{0}^{-}$

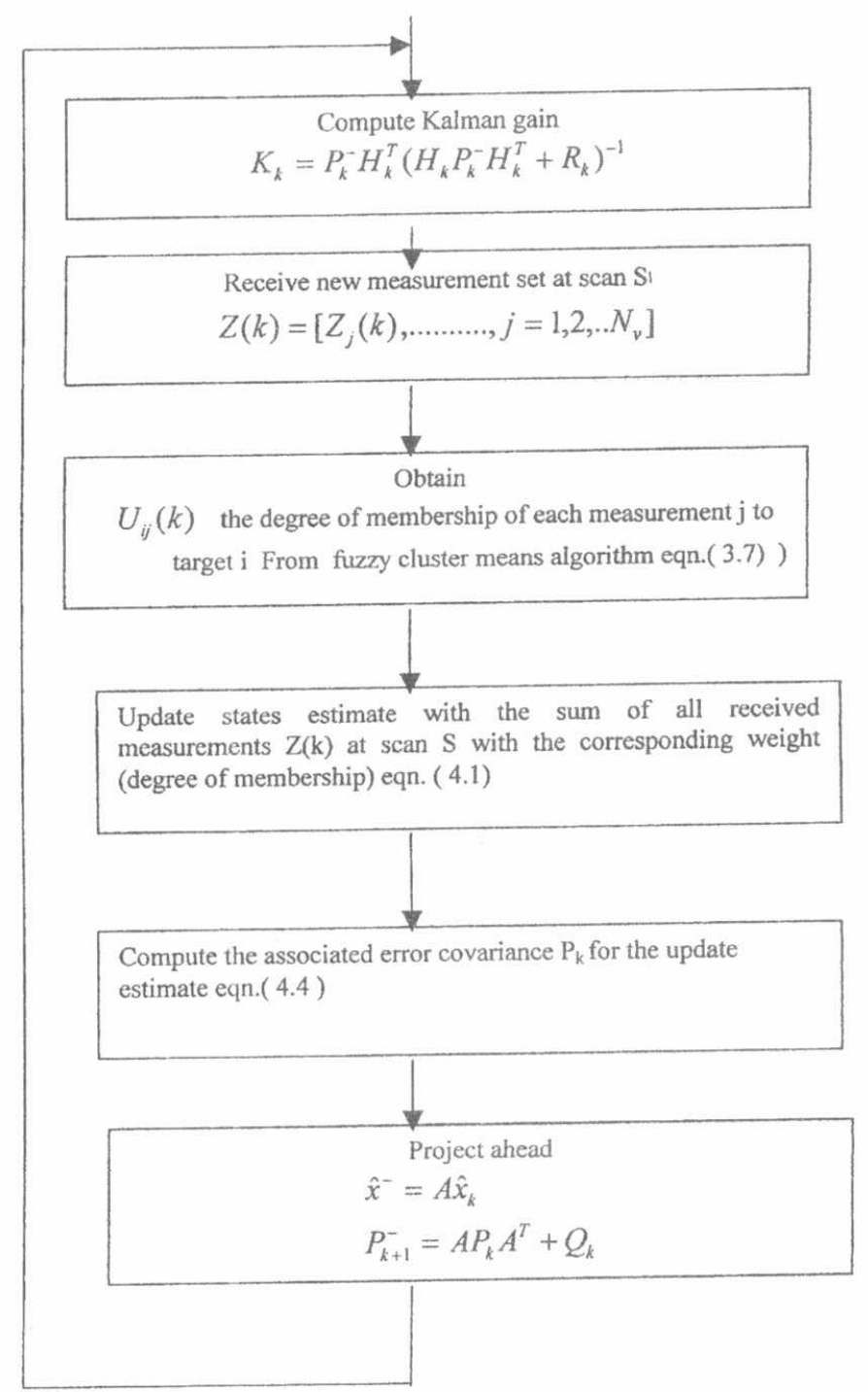

Fig.1 flow diagram of the proposed Fuzzy Data Association Filter (FDAF)

\section{5-Simulation Results.}

To assess the performance of the proposed (FDAF), we suppose a test scenario of two targets (close together) moving with constant course and speed until time $t=30$ $\mathrm{sec}$ when they maneuver highly in $\mathrm{x}$ and $\mathrm{y}$ direction with acceleration inputs $(10 \mathrm{~g}$, $10 \mathrm{~g})$ for target 1 , and $(10 \mathrm{~g},-10 \mathrm{~g})$ for target 2 until $t=50 \mathrm{sec}$ then they made another maneuver but in the reverse directions i.e. with acceleration inputs $(-10 \mathrm{~g},-10 \mathrm{~g})$ for 
target 1 , and $(-10 \mathrm{~g}, 10 \mathrm{~g})$ for target 2 until $t=80 \mathrm{sec}$, the two targets again move with constant course and acceleration until $t=100 \mathrm{sec}$. The initial conditions of the two maneuvering targets are as follows:

Target1: $\left(x_{1}(0)=50,000 \mathrm{~m}, x_{1}(0)=-500 \mathrm{~m} / \mathrm{sec}, y_{1}(0)=20500 \mathrm{~m}, \dot{y}_{1}(0)=0\right)$

Target2: $\left(x_{2}(0)=50,000 \mathrm{~m}, . x_{2}(0)=-520 \mathrm{~m} / \mathrm{sec}, y_{2}(0)=21000 \mathrm{~m}, \dot{y}_{2}(0)=0\right)$

The error of measurement for target 1(tg1): $\left(\sigma_{x, y}=50 \mathrm{~m}, \sigma_{\dot{x}, \dot{y}}=25 \mathrm{~m} / \mathrm{sec}\right)$.

The error of measurement for target 2(tg2): $\left(\sigma_{x, y}=100 \mathrm{~m}, \sigma_{\dot{x}, \dot{y}}=25 \mathrm{~m} / \mathrm{sec}\right)$.

The sampling time $T=1 \mathrm{sec}$.

A Monte Carlo simulation of 50 runs has been obtained and the results are depicted, Fig. 2 shows the true and the measured target trajectories, Fig. 3 depicts the degree of membership of measurements 1,2 to target 1 during $100 \mathrm{sec}$. The degree of membership of measurement 1,2 to target 2 is in Fig. 4 .

The results of tracking the two maneuvering targets by applying the proposed (FDAF) and by applying the standard Kalman filter (KF) are depicted in Fig. 5 and Fig.6 respectively. The True and estimated velocity track in X-direction for $(\operatorname{tg} 1)$ and (tg2) are depicted in Fig.7 and Fig.8. respectively. The plots of the root mean square position and velocity error in X-direction for $(\operatorname{tg} 1)$ and $(\operatorname{tg} 2)$ are depicted in Fig.9 and Fig.10 respectively, (the plot in $Y$-direction are similar so it is omitted). It has been shown that the two trackers appear to be equally effective in the constant course of the target trajectories. However, during the maneuvering period the (FDAF) has a lower average error than the standard Kalman filter.

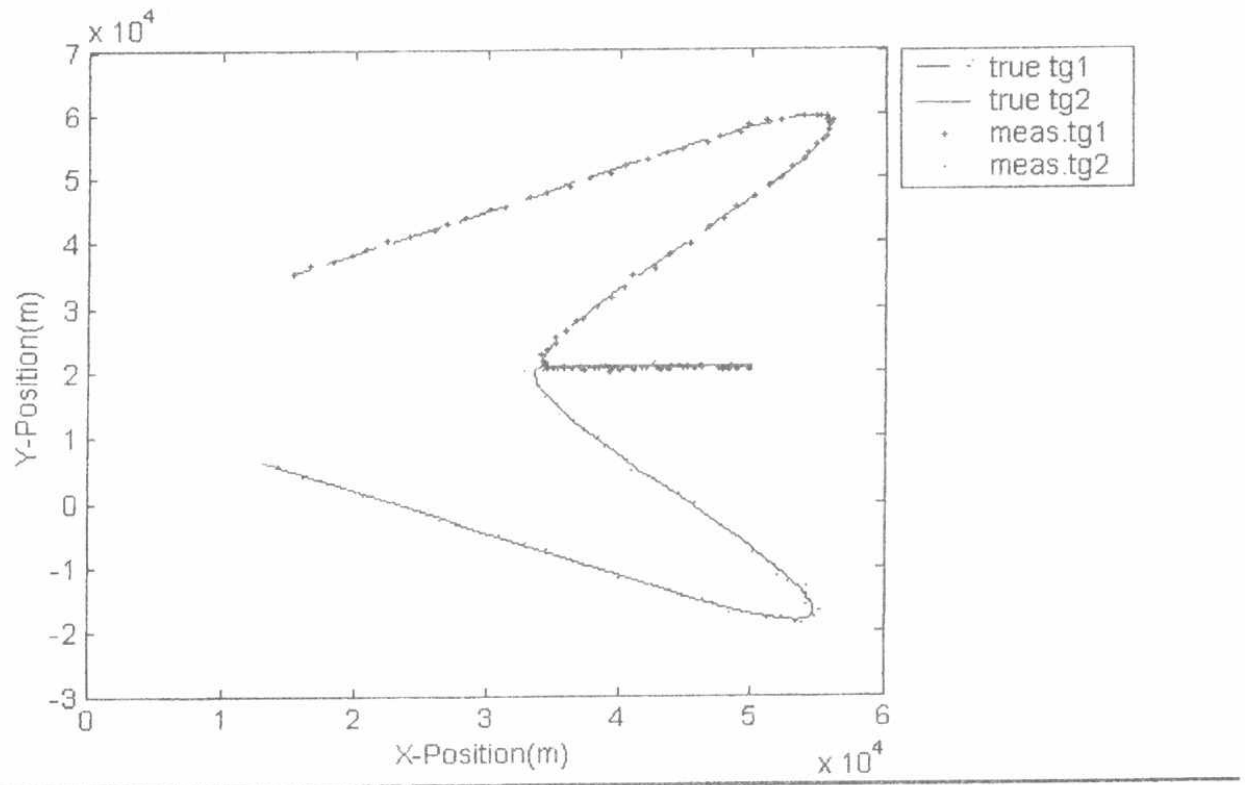

Fig. 2 True and measured target trajectories 

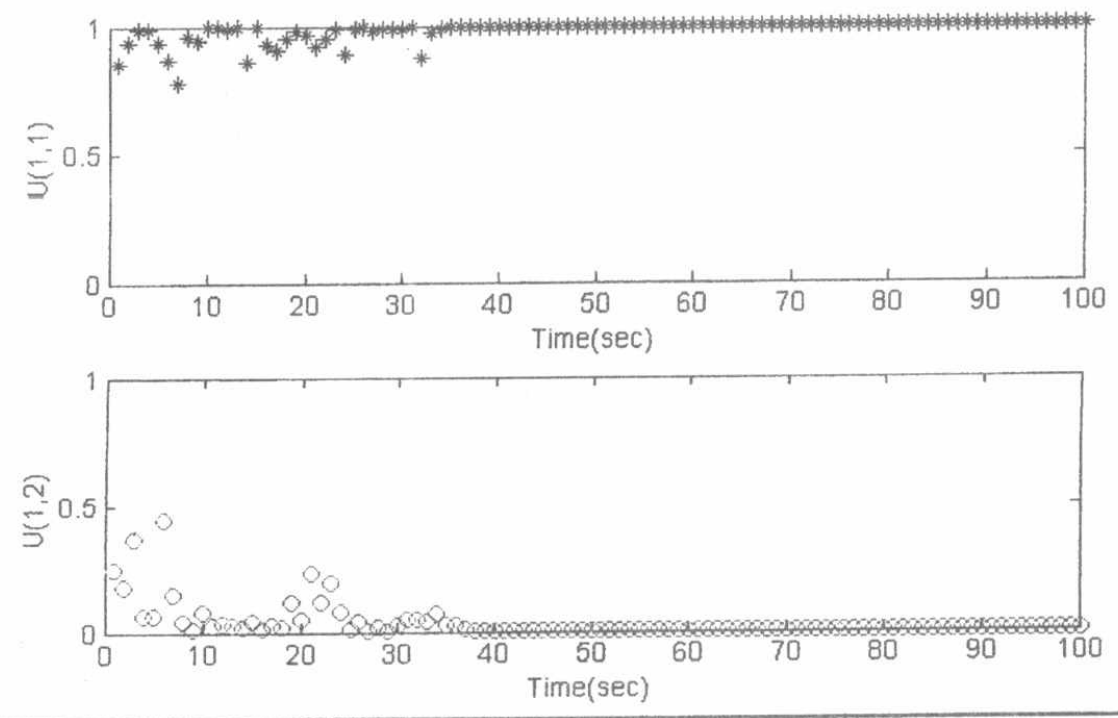

Fig. 3 The degree of membership of measurement $(1,2)$ to target 1
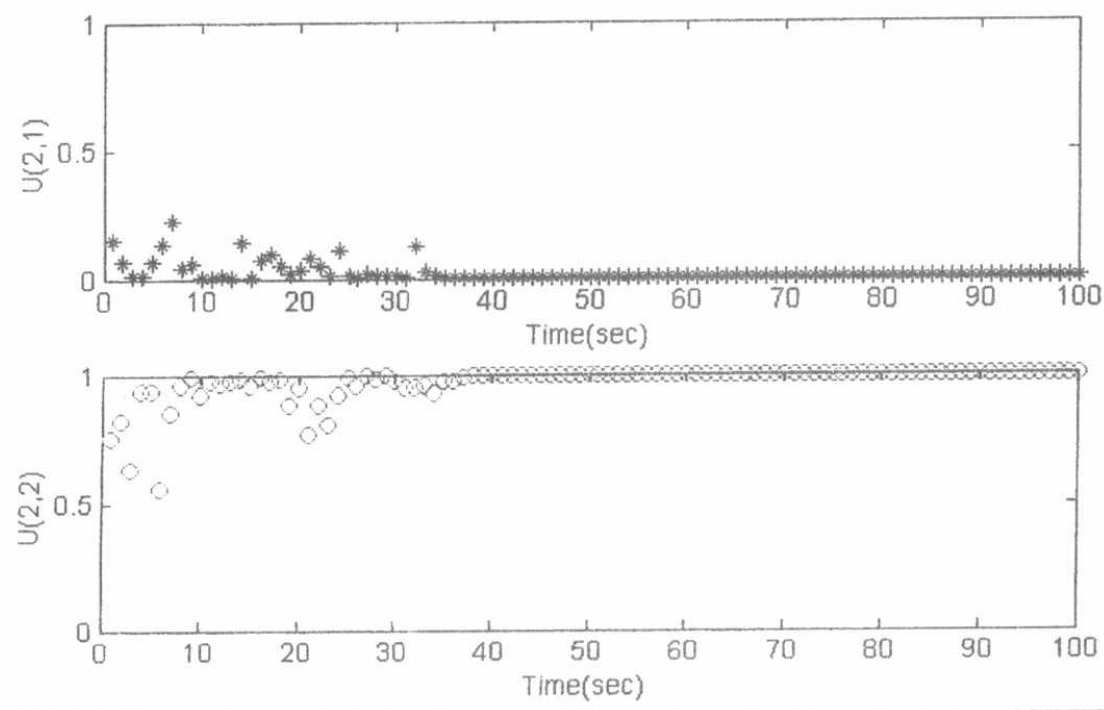

Fig. 4 The degree of membership of measurement $(1,2)$ to target 2 


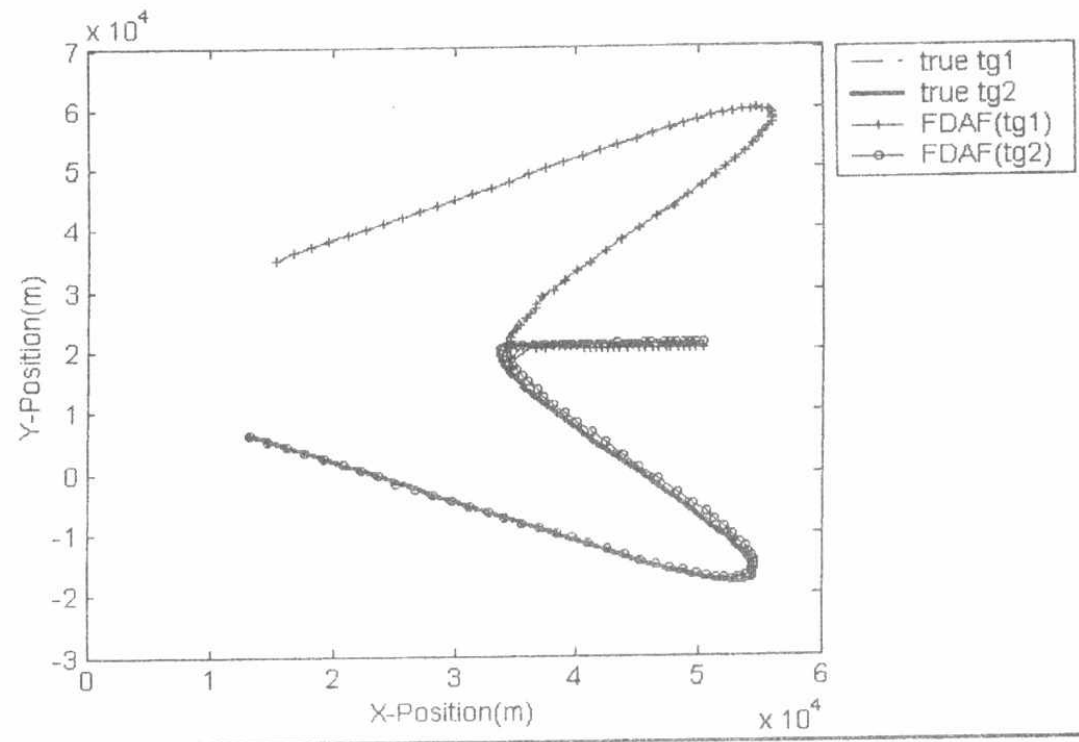

Fig.5 True and estimated target trajectories (FDAF).

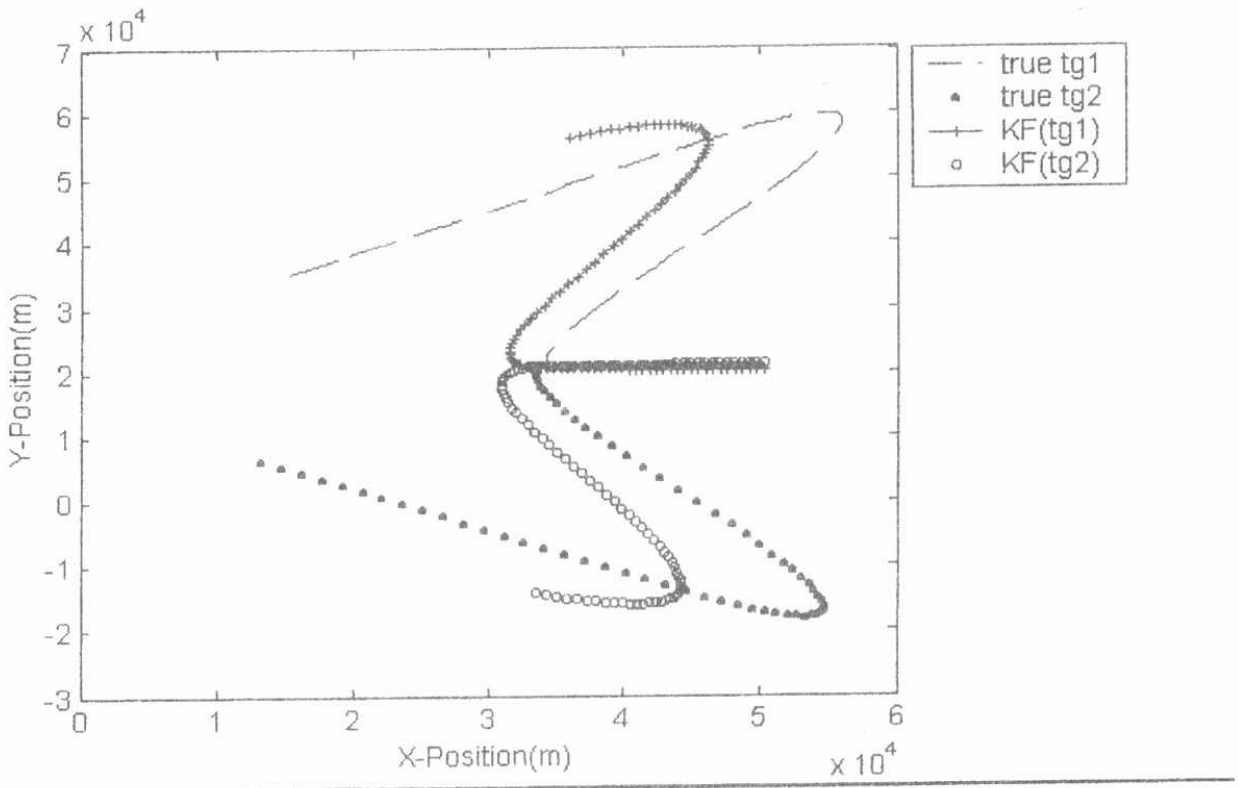

Fig.6 True and estimated target trajectories (K.F). 


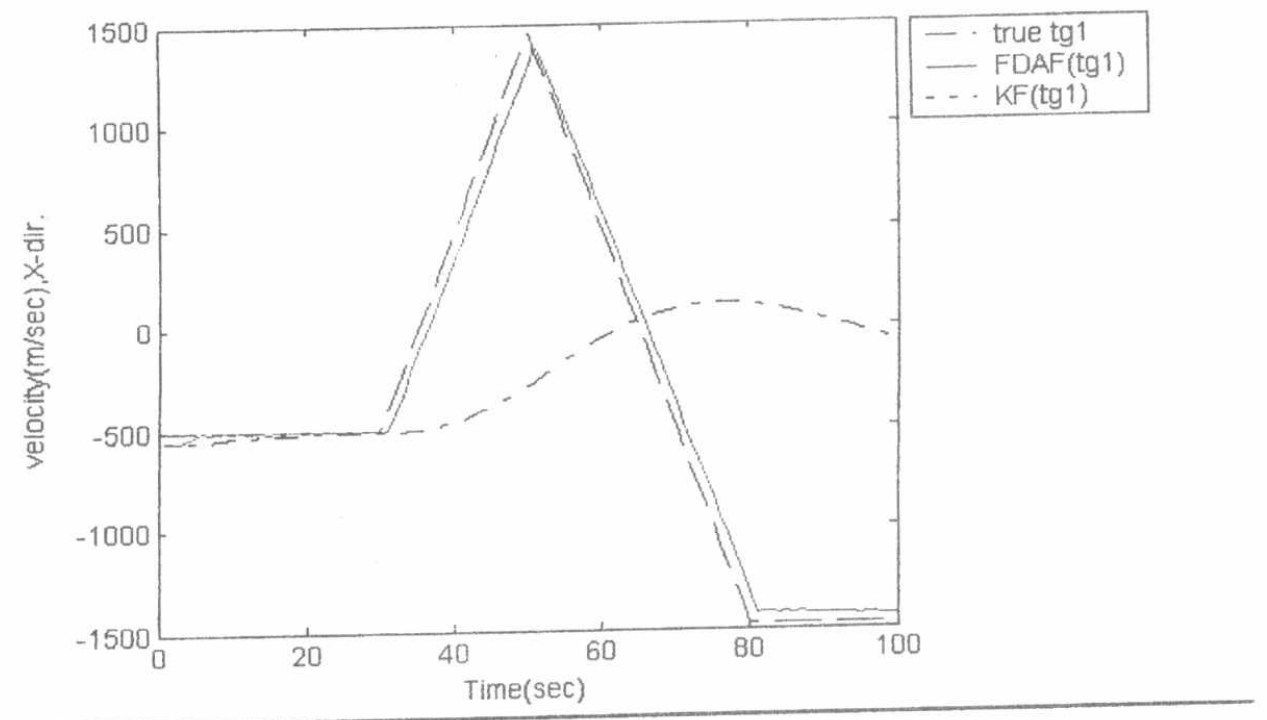

Fig. 7 True and estimated velocity track $(\operatorname{tg} 1)$

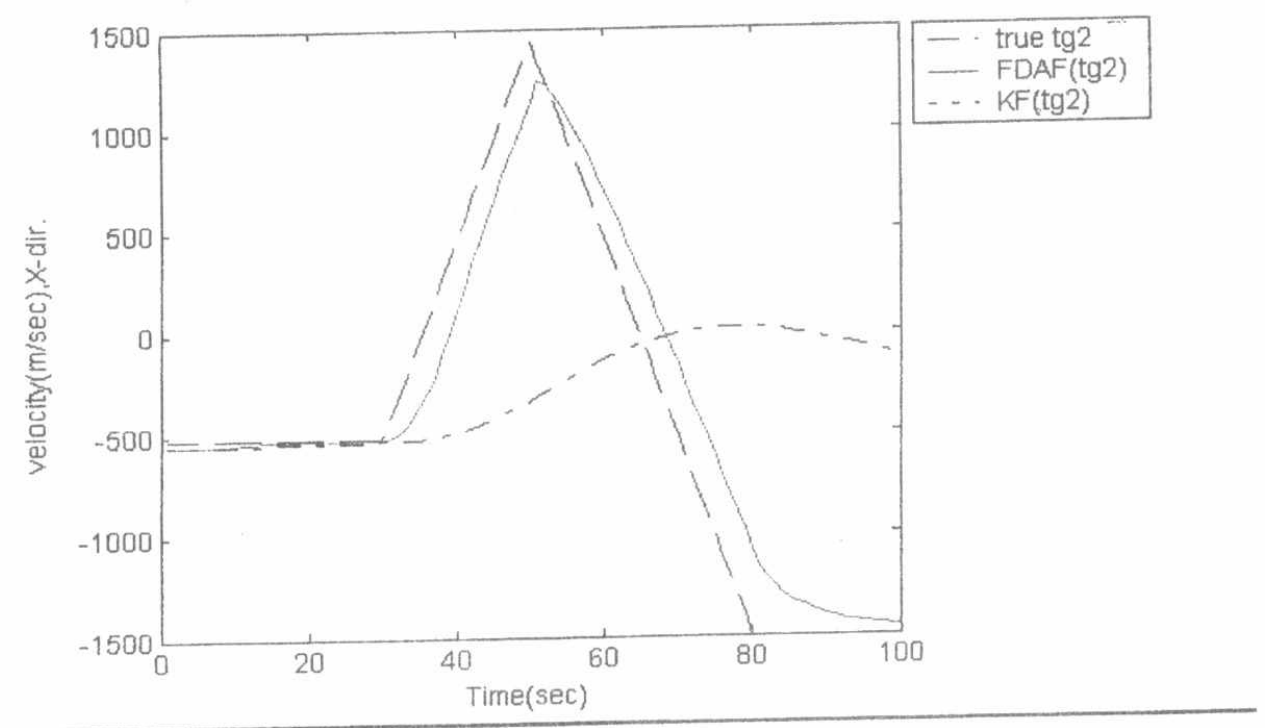

Fig. 8 True and estimated velocity track (tg2) 


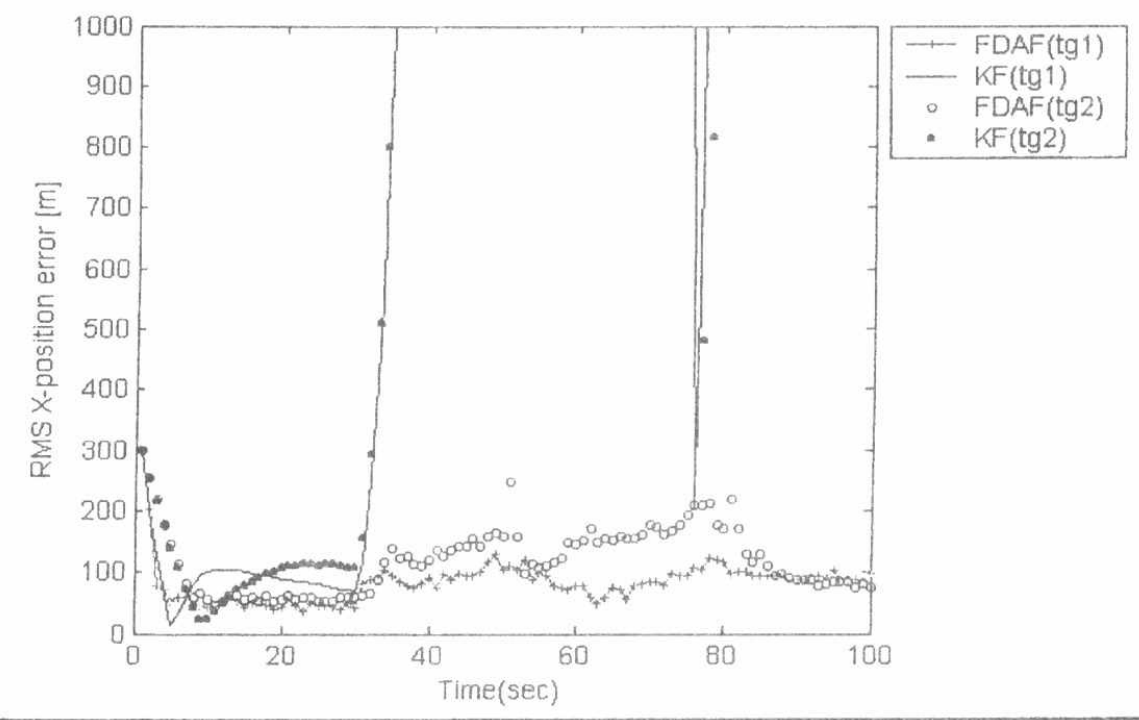

Fig.9 The root mean square $X$ - position error.

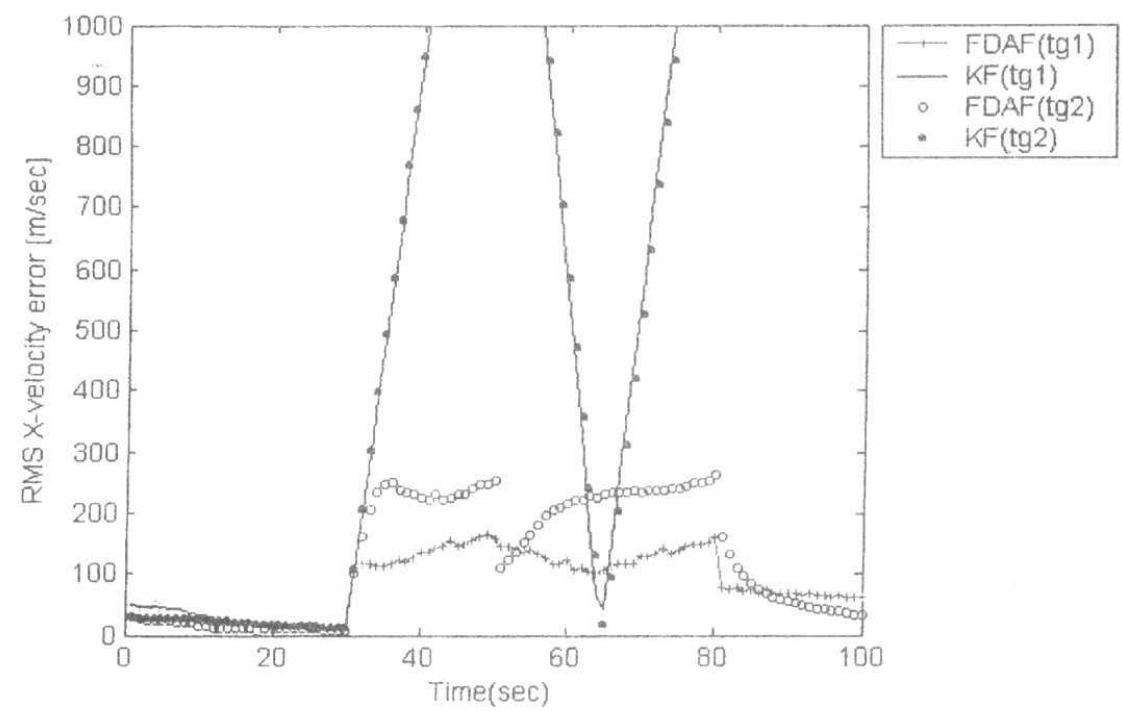

Fig.10 The root mean square $X$ - velocity error. 
It is worth noting that RMS of both position and velocity error are strongly affected by the noise level of measurements of the targets $\left(\sigma 1_{x, y}, \sigma 2_{x, y}\right)$. Table 1 shows this effect, the values of the RMS position and velocity errors for both targets $(\operatorname{tg} 1, \operatorname{tg} 2)$ in $\mathrm{X}$-direction, and in Y-direction, taking the noise level for both targets as a parameter is tabulated. It is recommended to cosider that the noise levels of both targets are equal.

Table 1

\begin{tabular}{|c|c|c|c|c|c|c|}
\hline$\sigma 1_{x, y}, \sigma 2_{x, y}[m]$ & $\underline{50,50}$ & $\underline{75,75}$ & 100,100 & 110,110 & 120,120 & 130,130 \\
\hline Av. RMS X-position error $\operatorname{tg} 1[\mathrm{~m}]$ & 54.7 & 63.6 & 81.4 & 102.4 & 105.1 & 123.5 \\
\hline Av.RMS-X position error tg2[m] & 55.6 & 64.0 & 83.2 & 91.7 & 108.9 & 130.8 \\
\hline Av. RMS-Y position error $\operatorname{tg} 1[\mathrm{~m}]$ & 53.1 & 61.6 & 78.7 & 99.4 & 100.1 & 756.5 \\
\hline Av. RMS-Y position error tg2 $[\mathrm{m}]$ & 53.0 & 60.6 & 79.9 & 86.6 & 101.2 & 757.5 \\
\hline $\begin{array}{l}\text { Av. RMS X-velocity error } \\
\operatorname{tg} 1[\mathrm{~m} / \mathrm{sec}]\end{array}$ & 69.1 & 71.9 & 66.6 & 81.1 & 67.2 & 93.5 \\
\hline $\begin{array}{l}\text { Av. RMS-X-velocity error } \\
\operatorname{tg} 2[\mathrm{~m} / \mathrm{sec}]\end{array}$ & 68.9 & 70.9 & 67.2 & 64.3 & 68.2 & 94.6 \\
\hline $\begin{array}{l}\text { Av. RMS-Y velocity error } \\
\operatorname{tg} 1[\mathrm{~m} / \mathrm{sec}]\end{array}$ & 70.9 & 72.6 & 67.2 & 82.6 & 67.7 & 70.1 \\
\hline $\begin{array}{l}\text { Av. RMS-Y velocity error } \\
\text { tg2 }[\mathrm{m} / \mathrm{sec}]\end{array}$ & 69.9 & 71.3 & 65.9 & 64.1 & 67.1 & 69.8 \\
\hline
\end{tabular}

The results indicate that the proposed (FDAF) achieves reasonable performance even in the presence of high level of measurement noise.

\section{6- Conclusion.}

A new tracking algorithm (FDAF) for tracking maneuvering multitarget tracking is presented in this paper. The algorithm incorporates all the observations within the gate of the predicted target state to update the state estimate using a membershipweighted sum of innovations obtained by Fuzzy cluster means algorithm. The major contribution of this work is the development of algorithm, which solves the interrelated tasks of data association and state estimation in one combined algorithm. The results show the effectiveness of the proposed algorithm to reduce the root mean square (position error, velocity error) in high maneuvering targets, and high level of measurement noise.

\section{References}

[1] S.S Blackman, Multiple Target Tracking with radar application, Norwood, MA, Artech House, 1986.

[2] D.L.Hall, Mathematical Techniques in multisensor data fusion, Norwood, MA, Artech House, 1992.

[3] Y.Bar-Shalom and T.Fortman, Tracking and Data Association, Academic Press, , 1988.

4] Y.Bar-Shalom and X. Rong Li, Estimation and Tracking : Principles, Techniques, and Software, Artech House, Boston, London, 1993.

[5]Y.Bar-Shalom, Multitarget Multisensor Tracking:Applications and Advances, Vol I Norwood, MA, Artech House, 1990. 
[6] Y.Bar-Shalom, Multitarget Multisensor Tracking:Applications and Advances, Vol II Norwood, MA, Artech House, 1992.

[7]D.Sengupta,et al., Neural Solution to the Multiple Target Tracking Data Association Problem, IEEE Tranasactions on Aerospace and Electronic Systems, Vol.AES-25, 1989, .96-108.

[8]Bar-Shalom, Y., and Birmiwal, K., Consistency and robustness evaluation of the PDAF for target tracking in a cluttered environment, Automatica, VOL.19, 1983 No 4, 431-436

[9]Bar-Shalom, Y., and Tse, E., Tracking in a cluttered environment with probabilistic data association, Automatica, 11, Sept.1975, 451-460.

[10]Chang,K.C, Chong, and Bar-Shalom, Joint probabilistic data association in distributed sensor networks, IEEE Transactions on Automatic Control, AC-31, 10(Oct1986), 889-897.

[11] Reid,D.B, An algorithm for tracking multiple targets, IEEE Transactions on Automatic Control, AC-24, 12(Dec1979), 843-854.

[12]B.Zhou, N.K.Bose, An efficient algorithm for data association in multitarget tracking, IEEE Tranasactions on Aerospace and Electronic Systems, Vol.31, No1, Jan1995, 458-468.

[13]H.M.Sun. and S.M.Chiang, Manoeuvring target tacking algorithm for a radar system, INT.J.Systems, 1989, Vol20, No10, 1801-1811.

[14]H.M.Sun. and S.M.Chiang, Manoeuvring multitarget tracking method in cluttered environment, INT.J.Systems, 1990, Vol.21, No12, .2469-2487.

[15]D.Lerro and Bar-Shalom, Interacting multiple model tracking with target amplitude feature", IEEE Tranasactions on Aerospace and Electronic Systems, Vol.29, No.2, April 1993, 494-508

[16]K.Birmiwal and Bar-Shalom, On Tracking a maneuvering target in clutter, IEEE Tranasactions on Aerospace and Electronic Systems, Vol.AES-20, No.5, Sept1984, 635-645.

[17]Arie Berman, Amnon Hammer, False alarm effects on estimation in multitarget trackers, IEEE Tranasactions on Aerospace and Electronic Systems, Vol.27, No.4, July1991, 675-681

[18]A.Houles, Bar-Shalom, Multisensor tracking of a maneuvering target in clutter, IEEE Tranasactions on Aerospace and Electronic Systems, Vol.AES-25, No.2, March1989, 176-189.

[19] Xiao and Bar- Shalom, Multiple-Model Estimation with Variable Structure, IEEE Tranasactions on Automatic Control, Vol.41, No.4, April 1996, 478-493. 
[20]Ashraf Mamdouh Aziz,Murali and Roberto, Fuzzy logic data correlation in multisensor-multitarget tracking systems, Signal Processing, Vol 76, No.2, July1999, 195-209.

[21]O.Hossam, M.Farooq, and T.Quach, Fuzzy logic approach to data association, Proceedings of SPIE, Vol.2755, 1996, 313-321.

[22]R.P. Singh and W.H. Balley, Fuzzy logic applications to multisensor-multitaret corrlation, IEEE Tranasactions on Aerospace and Electronic Systems, Vol.33, NO3, July1997, 752-769.

[23]Michael P. WINDHAM, Geometrical Fuzzy Clustering Algorithms, Fuzzy Sets and Systems 10 (1983) 271-279.

[24]B.Zhou, N.T. Bose, An efficient algorithm for data association in multitarget tracking, IEEE Tans.Aerospace Electron. System 31(1) (January 1995) 458-468.

[25]Wide, A fuzzy approach to multi-sensor data fusion for quality profile classification, Proceeding of the IEEE/SICE/RSJ International conference on Multisensor Fusion and Integration for Intelligent Systems, 1996, 215-221.

[26]J.C. Bezdek, Pattern Recognition with Fuzzy Objective Function Algorithms, Plenum Press, New York, 1981.

[27]J.Smith, A fuzzy logic multisensor association algorithms, Proc. SPIE 3068(1997)76-87.

[28]L.A Zadeh, Fuzzy sets, Inform. And Control 8 (1965) 338-353.

[29]R.J. Hathaway, G. Rogers, J.C. Bezdek, W.Pedrycz, Fusing heterogenous fuzzy data for clustering. Proc. SPIE 3068 (1997) 559-568.

[30]Murali, Bar-Shalom, IMM estimation for multitarget-multisensor airtraffic surveillance, proceeding of the IEEE. Vol.85.NO.1.January, 1997, 80-93.

[31]Seong, Jang, Imroved Kalman filter design for Three-dimensional radar tracking, IEEE Tranasactions on Aerospace and Electronic Systems, Vol.37, N0 2, April 2001, 728-738.

[32] Jeong, Ho park, Amultiple- target tracking filter using data association based on MAP approach, IEICE Trans. Fundamentals, Vol.E 83-A, No 6 June 2000., 12031210

[33]Anwar, Bahnasawi, Fuzzy solution for data association in multisensor-multitarget tracking in a cluttered environment, submitted for publication 2003. 